\title{
Self-consistent field theory of polymer-ionic molecule complexation
}

\author{
Issei Nakamura ${ }^{\text {a) }}$ and An-Chang Shib) \\ Department of Physics and Astronomy, McMaster University, 1280 Main Street West, Hamilton, \\ Ontario L8S 4L8, Canada
}

(Received 14 March 2010; accepted 27 April 2010; published online 17 May 2010)

\begin{abstract}
A self-consistent field theory is developed for polymers that are capable of binding small ionic molecules (adsorbates). The polymer-ionic molecule association is described by Ising-like binding variables, $C_{i}^{(a)}(k \Delta)(=0$ or 1$)$, whose average determines the number of adsorbed molecules, $n_{\mathrm{BI}}$. Polymer gelation can occur through polymer-ionic molecule complexation in our model. For polymer-polymer cross-links through the ionic molecules, three types of solutions for $n_{\mathrm{BI}}$ are obtained, depending on the equilibrium constant of single-ion binding. Spinodal lines calculated from the mean-field free energy exhibit closed-loop regions where the homogeneous phase becomes unstable. This phase instability is driven by the excluded-volume interaction due to the single occupancy of ion-binding sites on the polymers. Moreover, sol-gel transitions are examined using a critical degree of conversion. A gel phase is induced when the concentration of adsorbates is increased. At a higher concentration of the adsorbates, however, a re-entrance from a gel phase into a sol phase arises from the correlation between unoccupied and occupied ion-binding sites. The theory is applied to a model system, poly(vinyl alcohol) and borate ion in aqueous solution with sodium chloride. Good agreement between theory and experiment is obtained. (C) 2010 American Institute of Physics. [doi:10.1063/1.3430745]
\end{abstract}

\section{INTRODUCTION}

The miscibility of neutral polymers and polyelectrolytes with ionic molecules in solvents has attracted much interest in polymer science over the last decades. ${ }^{1}$ In particular, phase separation induced by complexation between polymers and ionic molecules ${ }^{2-10}$ is widely studied in experiments ${ }^{11-14}$ and theory. ${ }^{15-18}$ Noncovalent intermolecular interactions such as hydrogen bonding, dispersion, and electrostatic forces are often employed to bind smaller molecules onto polymers (adsorbents). For example, functionalized sidechains of polymers are capable of capturing relatively smaller molecules through these weak forces. ${ }^{19,20}$ This association is typically sensitive to temperature, $p \mathrm{H}$, and salt concentration. The reversibility of the complexation processes can be utilized for various potential applications in chemical engineering or supramolecular science to fabricate functional materials such as hydrogel. ${ }^{21}$

Adsorption phenomena are often described by the Langmuir model, where an adsorbate molecule (B) is adsorbed onto an unoccupied binding site $(\mathrm{P})$ to form an adsorbed complex (BP). The Langmuir model is based on the following assumptions: (1) the adjacent molecules adsorbed on the binding sites are mutually noninteracting, (2) the surface at which there are binding sites is geometrically uniform (i.e., not corrugated), and (3) each binding site is capable of adsorbing a single molecule. The number of adsorbed molecules is determined by the chemical equilibrium between

\footnotetext{
${ }^{a)}$ Electronic mail: nakamur@caltech.edu. Present address: Division of Chemistry and Chemical Engineering, California Institute of Technology, Pasadena, California 91125, USA.

${ }^{b)}$ Electronic mail: shi@mcmaster.ca.
}

nonadsorbed (free) adsorbates in bulk phase and adsorbed adsorbates. In this case, the association equilibrium is written as $\mathrm{B}+\mathrm{P} \rightleftarrows \mathrm{BP}$ (monocomplexation) with an equilibrium constant, $K_{\text {eq. }}$. The Langmuir equation is then given by

$$
[\mathrm{BP}]=\frac{[\mathrm{BP}]_{\max } K_{\mathrm{eq}}[\mathrm{B}]}{1+K_{\mathrm{eq}}[\mathrm{B}]},
$$

where $[\mathrm{BP}]_{\max }$ is the maximum value of $[\mathrm{BP}]$ as $[\mathrm{B}] \rightarrow \infty$. It should be noticed that in general, an adsorbate molecule may be capable of binding to two (or more) binding sites. In this case, further complexation between BP and P can lead to interpolymer or intrapolymer association (dicomplexation) or gelation, $\mathrm{BP}+\mathrm{P} \rightleftarrows \mathrm{BP}_{2}$.

For polyelectrolytes, adsorption of monovalent and divalent counterions on charged sites occurs at low temperatures, effectively neutralizing the polyelectrolytes. In this case, a polyelectrolyte chain may collapse because the repulsive force between charged sites is weakened. ${ }^{22-24}$ Upon further addition of the divalent salts, the charges on the polyelectrolyte chain can be reversed as a result of an excess number of condensed counterions, corresponding to the charge inversion in colloidal science. ${ }^{25}$

To characterize the Coulombic attractive force for adsorptions at a temperature, $T$, the Bjerrum length, $l_{B}$ $=e^{2} / 4 \pi \varepsilon k_{B} T$, and Coulomb coupling constant (or dimensionless inverse temperature), $\Gamma=l_{B} / a$, are often employed. Here, $e, \varepsilon, k_{B}$, and $a$ denote the unit charge, dielectric constant, Boltzmann constant, and the distance between charged molecules, respectively. When $\Gamma>1$, the complexation between charges occurs, indicating a strong coupling limit of the system. However, it is known that the Poisson-Boltzmann equation (PBE), a classical mean-field theory, fails to describe the 
binding of the charges, ${ }^{26}$ even though the electrostatic screening of charged sites is included in the PBE. Thus, an explicit description of ion binding on polymer is needed in theory.

Complexation of polyvinyl alcohol (PVA) $)^{3,5,11,16,17}$ or hydroxypropyl guar (HPG) ${ }^{6,10}$ and borate ions $\left(\mathrm{B}(\mathrm{OH})_{4}^{-1}\right)$ is another prominent example of molecular binding interactions. Isotactic diol groups of these hydrosoluble polymers are capable of binding borate ions in aqueous solution, causing the neutral polymers to become polyelectrolytes after complexation occurs. The degree of ionization is typically sensitive to temperature, $p \mathrm{H}$, and ionic strength. The charge fraction of the polymers due to bound borate ions is determined by the chemical equilibrium of the adsorption on diol groups. Furthermore, cross-linking formation between polymers can be induced by the two binding sites of a single borate ion. This reaction leads to thermoreversible gels at high concentrations of the polymers under certain conditions. In dilute solutions, conformational changes of a single polymer can occur through intramolecular cross-links. A demixing of the polymers can also be induced by increasing the number of cross-links. Then, precipitated polymers are obtained, forming a dense gel. In semidiluted solutions, an opaque gel is observed with or without the demixing.

In general, the phase instability of a system driven by the complexation between polymer and ionic molecules is determined by the competition between enthalpy gain and entropy loss due to the adsorption of adsorbates. At the same time, the free energies of other nonadsorbed molecules such as salt ions cannot be ignored. The free energy balance typically leads to a closed-loop structure of phase diagrams. ${ }^{15}$ Moreover, a gelation through polymer-ion-polymer association may largely modify the total free energy of the system because of entropy loss due to an increase in local monomer concentrations. In other words, adsorption equilibriums of the monocomplexation and dicomplexation can be strongly correlated. In this case, the nature of the complexation phenomena should be appropriately incorporated into theoretical models for the modification of the Langmuir equation [Eq. (1)] so that all of the effects associated with the free energy are self-consistently correlated through existing components in the systems. The free energy is, however, often written as a function of only a single variable such as polymer concentration. In this perspective, the association of supramolecular diblock copolymers is studied using a self-consistent field theory (SCFT) ${ }^{27}$ For the system of polymers and ions, further development of the theory with the polymer-ion association is also needed.

In this paper, a generic model for a class of flexible polymers exhibiting complexation with small ionic molecules is constructed. The polymers have binding sites where small ionic molecules can be adsorbed. A SCFT of the system is developed to include the specific-binding interaction. We remark that the model can be regarded as an extension of the weakly charged polyelectrolyte theories by Shi and Noolandi ${ }^{28}$ or Wang et l. $^{29}$ and an analytically solvable model of rigid macromolecules by Nakamura and Shi. ${ }^{30} \mathrm{~A}$ notable difference between the present and previous models

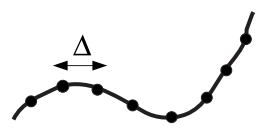

(a)

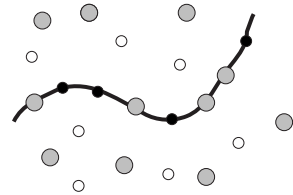

(b)
FIG. 1. Schematic descriptions of the system. (a) The binding sites are uniformly distributed on the adsorbent polymers with an interval, $\Delta$. (b) Ionic molecules (shaded circles) are specifically adsorbed on the polymers. The empty circles represent salt ions. The $k$ th binding site of the $i$ th polymer lies at $k \Delta$ from the 0 th site. In this paper, the polymers are indicated by the super/subscripts "a" on their variables.

is that the complexation equilibrium between polymer and small ionic molecule is explicitly taken into account in this paper.

In Sec. II A, mean-field equations and free energy are derived from the model. A generic understanding of the complexation phenomena is then obtained. To examine the applicability of the theory, PVA and borate ion in aqueous solution with sodium chloride $(\mathrm{NaCl})$ are chosen as a model system. These results are used to discuss binding isotherms of adsorbate molecules in the homogeneous phase (Secs. II B and II C). In Sec. II E, a mean-field potential for a gelation of polymers is introduced. Spinodal lines are obtained to study the instability of homogeneous phase. It should also be noted that we do not fix the concentrations of any species, and hence a multicomponent system is considered. In Secs. III A and III B, the correlation between occupied and unoccupied binding sites on the polymers is discussed for an improvement of the binding isotherm. In this case, the Langmuir equation is modified to reflect the effect of the correlation. The results are compared with an experiment for an intraconnected polymer in Sec. III C. In Sec. III D, the critical degree of conversion is calculated using the model in order to evaluate sol-gel transitions. Good agreement is indicated between the theory and experimental observations in all cases. Discussion and conclusions are presented in Sec. IV.

\section{MEAN-FIELD THEORY FOR THE COMPLEXATION WITHOUT POLYMER-ION CROSS-LINKING (MODEL A)}

\section{A. Model A}

Our model system contains flexible homopolymers (adsorbents), ionic molecules (adsorbates), salt ions, and neutral solvents in a volume, $V$. The polymers are capable of binding the ionic molecules at binding sites along the chains. The complexation between the polymer and ionic molecule is schematically illustrated in Fig. 1. The binding reaction is also described as

$$
\mathrm{B}+\mathrm{P} \rightleftarrows \mathrm{BP},
$$

where B, P, and BP denote the free nonadsorbed adsorbate molecule, unoccupied ion-binding site, and occupied ionbinding site with the adsorption, respectively. The equilibrium constant of this monocomplexation is expressed as $K_{\mathrm{eq}}$. The total numbers of polymers, binding sites on a polymer, ionic molecules, salt ions, solvents, and salt ion species are denoted as $N_{a}, M_{a}, N_{-}, N_{j}, N_{v}$, and $I$ respectively. We define 
an Ising-like binding variable for the $k$ th binding site of the $i$ th polymer,

$$
C_{i}^{(a)}(k \Delta)= \begin{cases}1 & \text { (for adsorption) } \\ 0 & \text { (for nonadsorption })\end{cases}
$$

This indicates that a single ionic molecule is adsorbed on the $k$ th binding site if $C_{i}^{(a)}(k \Delta)=1$. Thus, the number of adsorbed

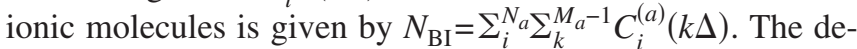
tailed partition function of the model is given in Appendix A. A functional form of the free energy and the mean-field equation of the system can be derived from the partition function using a standard technique of the SCFT.

\section{B. Homogeneous phase}

In this section, the effect of ion binding on the free energy of homogeneous phase is discussed using uniform solutions to the mean-field equations, Eq. (A19). The detailed derivation of the free energy is given in Appendix B.

The free energy of the system [Eq. (B9)] can be cast into (the standard Flory-Huggins free energy $)+(\Delta F$ =free energy change due to the binding interaction),

$$
\Delta F=\frac{-M_{a} n_{a}}{Z_{a}} \ln \left(1+g K_{\mathrm{eq}}\right),
$$

where $Z_{a}$ is the number of monomers of a homopolymer.

In many experimental conditions, $g K_{\text {eq }}<1$ is fulfilled. In this case, Eq. (4) is expanded to the series,

$$
\Delta F=-\gamma g K_{\mathrm{eq}}+\frac{\gamma}{2}\left(g K_{\mathrm{eq}}\right)^{2}+O\left(\left(g K_{\mathrm{eq}}\right)^{3}\right) .
$$

$g$ and $\gamma$ denote the overall concentrations of adsorbate molecules and binding sites, respectively. The first term indicates an attractive interaction between binding site and adsorbate molecule with a coupling constant, $K_{\text {eq }}$. However, a repulsive three-body interaction arises from the second term. When a binding site is occupied by an adsorbate molecule, other adsorbate molecules in the vicinity are expelled through the excluded-volume interaction. Thus, the second term implies a single occupancy on each binding site.

\section{Binding isotherm of model A}

Equations (B2)-(B8) can be applied to the experiments for binding isotherms of borate ion and PVA or HPG in aqueous solution. ${ }^{3,6}$ The values of $K_{\text {eq }}$ are estimated at $27.3\left[\mathrm{M}^{-1}\right]$ and $11.4\left[\mathrm{M}^{-1}\right]$ for the cases of PVA and HPG, respectively. Two neighboring monomers of PVA are identified as a statistical segment in the model. In the case that neighboring $-\mathrm{OH}$ groups are isotactic, the segment is considered a single binding site. This is because two $-\mathrm{OH}$ groups of a single borate ion participate in the complexation with the two $-\mathrm{OH}$ groups of the segment. Moreover, experimental observations indicate that there are two occupied binding sites per bound borate ion in dilute solution. ${ }^{3}$ This implies that a didiol type of complex between two binding sites and a single borate ion is formed on the polymers. The shrinkage of PVA due to cross-links upon addition of borate ions in aqueous solution is also studied using dynamic light

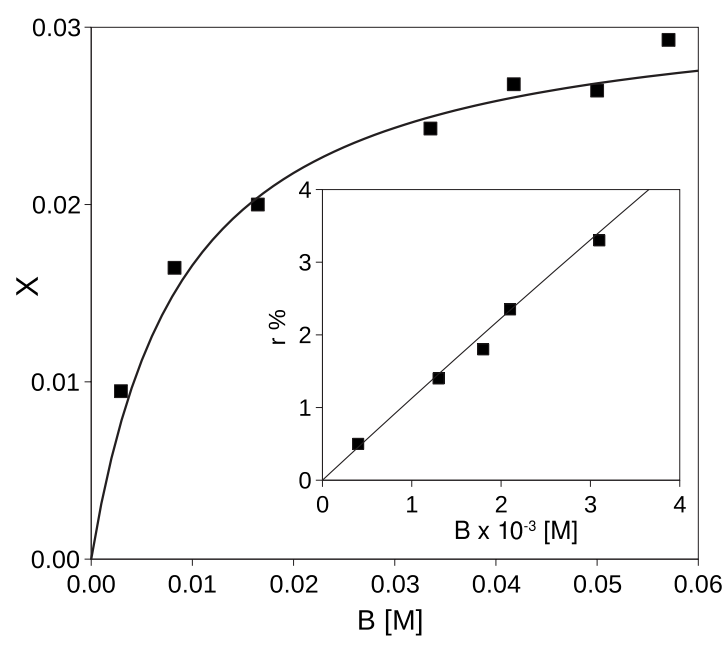

FIG. 2. Theoretical binding isotherm of borax to PVA. The $x$-axis and $y$-axis denote the free borax concentration and mol borax per monomol of PVA and X, respectively. $M_{a} / Z_{a}=0.127$ and $K_{\text {eq }}=27.3\left[\mathrm{M}^{-1}\right]$ are used, whereas the experimental $M_{a} / Z_{a}$ is 0.5 . The inset indicates a theoretical binding isotherm of borate for guaran with $K_{\mathrm{eq}}=11.4\left[\mathrm{M}^{-1}\right]$. The $x$-axis and $y$-axis denote the free borate concentration and the percentage of sugar units complexed with borate ions. Experimental data are reproduced from Refs. 3 and 6 with square dots.

scattering. ${ }^{8}$ Since model A does not include the cross-linking formation, a twofold difference of $\gamma$ between the theory and experiment is used to reproduce the experimental binding isotherms. Within this scope, the theoretical binding isotherm in Fig. 2 is in agreement with the result in Ref. 3. The applicability of the theory is also shown in the inset of Fig. 2 in the case of HPG. Further development of the model to include the effect of gelation is discussed in the following sections. Specifically, the twofold discrepancy of $\gamma$ between the theory and experiment will be improved in Sec. III C.

\section{Phase diagrams of the polymers and adsorbates in aqueous solution with monovalent salts}

In this section, spinodal lines are calculated using Eq. (B9). We consider PVA, borate $\left(\mathrm{B}(\mathrm{OH})_{4}^{-1}\right)$, and sodium chloride $(\mathrm{NaCl})$ in aqueous solution as a model system. In this paper, a system with a low ionic concentration is studied, and under this condition the Debye screening length is comparable to the distance between charged sites, allowing us to ignore a charge renormalization. ${ }^{17}$ In many experiments, borate is derived by a dissociation of borax $\left(\mathrm{Na}_{2} \mathrm{~B}_{4} \mathrm{O}_{7} \cdot 10 \mathrm{H}_{2} \mathrm{O}\right)$ in aqueous solutions. Here we denote the concentration of monovalent cations as $n_{+}$. The size of sodium chloride is ignored. The volumes of adsorbed and nonadsorbed ionic molecules are unchanged; thus, we set $\nu_{-}=\nu_{\mathrm{BI}} \equiv \nu_{B}$. In this case, Eq. (B9) leads to

$$
\begin{aligned}
\frac{\beta F}{V} \equiv f= & \lambda n_{a}\left(\rho_{0}-\nu_{a} n_{a}-\nu_{B} g\right)+\frac{n_{a}}{Z_{a}} \ln \left[\frac{n_{a}}{\left(1+g K_{\mathrm{eq}}\right)^{M_{a}}}\right] \\
& +g \ln g+n_{+} \ln n_{+}+\left(n_{+}-g\right) \ln \left(n_{+}-g\right) \\
& +\left(\rho_{0}-\nu_{a} n_{a}-\nu_{B} g\right) \ln \left(\rho_{0}-\nu_{a} n_{a}-\nu_{B} g\right),
\end{aligned}
$$

where the charge neutrality and the incompressibility are used. To study the instability of a homogeneous phase, spinodal lines are calculated by Eq. (6). ${ }^{31}$ It is noted that there are 


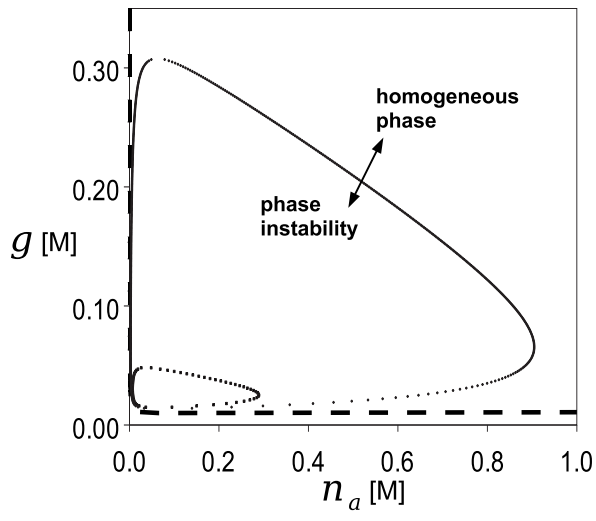

FIG. 3. Spinodal lines calculated from Eqs. (6) and (7) in $0.15[\mathrm{M}] \mathrm{NaCl}$ aqueous solution. The dashed and internal closed lines are obtained using $\Delta F$ of Eq. (5) to the first and second orders with $g K_{\mathrm{eq}}<1$, respectively. Phase instability occurs inside the closed dotted lines or above the dashed line.

three independent concentrations, $n_{a}, n_{+}$, and $g$. Hence, the spinodal lines are obtained by solving

$$
\left|\begin{array}{ccc}
\frac{\partial^{2} f}{\partial n_{a}^{2}} & \frac{\partial^{2} f}{\partial g \partial n_{a}} & \frac{\partial^{2} f}{\partial n_{+} \partial n_{a}} \\
\frac{\partial^{2} f}{\partial n_{a} \partial g} & \frac{\partial^{2} f}{\partial g^{2}} & \frac{\partial^{2} f}{\partial n_{+} \partial g} \\
\frac{\partial^{2} f}{\partial n_{a} \partial n_{+}} & \frac{\partial^{2} f}{\partial g \partial n_{+}} & \frac{\partial^{2} f}{\partial g^{2}}
\end{array}\right|=0 .
$$

Figure 3 shows the results with typical values of the parameters, $Z_{a}=1600, n_{+}=0.15[\mathrm{M}], M_{a} / Z_{a}=0.5, \lambda=8.64$ $\times 10^{-3}, K_{\text {eq }}=10\left[\mathrm{M}^{-1}\right], \rho_{0}=55.6[\mathrm{M}], \nu_{a}=4$, and $\nu_{B}=2 .{ }^{17,32}$ A closed-loop phase diagram is obtained from the model, indicating an instability of the homogeneous phase. The reentrance of the homogeneous phase is induced by varying the concentration of polymers or borates. In this case, the repulsive interaction due to the second order term in Eq. (5) is required to obtain the closed-loop structure of the phase diagram.

In experiments, instabilities of the homogeneous phases are observed in the case of PVA or HPG. ${ }^{12,16,17}$ The phase diagram in Fig. 3 indicates re-entrances into a homogeneous phase, as shown in Ref. 17. However, it gives rise to qualitative disagreement with the overall shape. That is, an observed dent structure of the phase diagram cannot be obtained in the present model. For any set of parameters in our calculations, the theoretical closed-loop diagrams are found to be in triangular form. Moreover, the lower threshold of the polymer concentration for the instability leads to a large discrepancy in magnitude, as the boundary of the experimental spinodal line is typically $n_{a} \geq 0.2[\mathrm{M}]$.

\section{E. The improvement of the phase diagram with a phenomenological potential for cross-linking}

It is widely known that the complexation between PVA (or HPG) and borate induces a gelation because borate is capable of binding to two diol units. This mechanism is, however, lacking in model A. Therefore, we introduce a

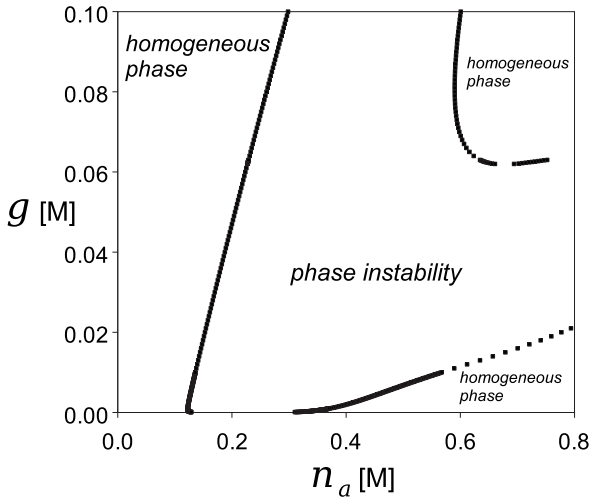

FIG. 4. Spinodal lines calculated using $V_{\text {gel }}\left(n_{s}\right)$. The set of parameters in Fig. 3 and $\alpha=0.6$ are used. Phase instability occurs inside the lines.

mean-field potential, $V_{\text {gel }}\left(n_{s}\right)$, for the interaction between adsorbed borate ion and unoccupied binding site of the polymers. ${ }^{33}$ This is written as

$$
\beta V_{\text {gel }}\left(n_{s}\right)=-\kappa \int d^{3} r n_{\text {up }}(\vec{r}) n_{\mathrm{BI}}(\vec{r})
$$

and

$$
\kappa=E K_{d},
$$

where $n_{\text {up }}$ denotes the concentration of unoccupied binding sites. $E$ is a didiol-borate complexation energy per dicomplex, and $K_{d}$ is a formation constant of the didiol-borate complexation $\left(\mathrm{BP}+\mathrm{P} \rightleftarrows \mathrm{BP}_{2}\right)$,

$$
K_{d}=\frac{\text { [didiol-borate concentration] }}{n_{\mathrm{up}} n_{\mathrm{BI}}} .
$$

Then, the mean-field interaction in the homogeneous phase using $n_{\mathrm{BI}}=K_{\mathrm{eq}} n_{\mathrm{up}} n_{-}$in Eq. (B7) can be cast into

$$
\frac{\beta V_{\mathrm{gel}}\left(n_{s}\right)}{V}=-\alpha \frac{n_{\mathrm{BI}}^{2}}{n_{-}},
$$

where $\alpha=E K_{d} / K_{\text {eq. }}$. Typical values of $\alpha$ and $K_{\text {eq }}$ are $\sim 0.1$ and $5 \sim 40\left[\mathrm{M}^{-1}\right]$, respectively. ${ }^{17}$ Figure 4 shows that the lower threshold of the polymer concentration with $\alpha=0.6$ shifts upward from that in Fig. 3 to the experimental value. Furthermore, the dent of the spinodal line is also obtained. These two pronounced improvements lead to qualitative agreement with the observation in the semidilute regime, $n_{a}>0.2[\mathrm{M}]$. Thus, the initial discrepancy is due to the absence of the cross-linking formation rather than the meanfield approximation (MFA).

\section{MODIFIED THEORY FOR POLYMER-ION CROSS- LINKING (MODEL B)}

\section{A. Model B}

In many systems, ion binding to polymers may induce molecular cross-linking, as found in the case of PVA with borate ions. Another example with an electrostatic nature is salt-bridging by divalent and monovalent counterions adsorbed on polyelectrolytes. ${ }^{33,34}$ In Sec. II E, the effect of the specific bindings on the phase stability of polymers is studied by incorporating the mean-field potential for cross-linking 
between bound ions and unoccupied binding sites of the polymers in the SCFT. However, Eqs. (8)-(11) do not modify the number of adsorbed borate ions in Eq. (B2). Therefore, the discrepancy in the binding isotherm discussed in Sec. II C cannot be improved. This is because the correlation between unoccupied binding sites and adsorbed borate ions is still lacking in model A.

In this section, as a generalization model A, the correlation between binding sites and ions is incorporated into the theory. When polymers adsorb molecules, which are capable of forming cross-links, unoccupied binding sites interact with the adsorbed molecules. This effect correlates with the conformation of the polymers via molecular association. In this case, intermolecular association is typically induced at high concentrations, whereas intramolecular association occurs at low concentrations. In terms of the SCFT, the mean potential of the interacting molecules expressed as an effective field, $\omega_{\text {up }}$, needs to be introduced to construct model B, which includes the correlation. Thus, the self-consistent fields, $n_{\text {up }}$ and $\omega_{\text {up }}$, are used to obtain the functional form of the partition function [Eq. (A12)] from Eq. (A11) with Eq. (8). That is, an additional functional integral, $\int \mathcal{D} n_{\text {up }} \Pi_{r} \delta\left(\hat{n}_{\text {up }}(\vec{r})-n_{\text {up }}(\vec{r})\right)$, is inserted in Eq. (A12). This can then be cast into

$$
\begin{aligned}
\int & \mathcal{D} n_{\mathrm{up}} \prod_{r} \delta\left(\hat{n}_{\mathrm{up}}(\vec{r})-n_{\mathrm{up}}(\vec{r})\right) \\
= & \int \mathcal{D} n_{\mathrm{up}} \mathcal{D} \omega_{\mathrm{up}} \exp \left[i \int d^{3} r \omega_{\mathrm{up}}(\vec{r})\left(n_{\mathrm{up}}(\vec{r})+n_{\mathrm{BI}}(\vec{r})\right)\right. \\
& \left.-i \sum_{i}^{N_{a}} \sum_{k=0}^{M_{a}-1} \int_{0}^{Z_{a}} d t \delta(t-k \Delta) \omega_{\mathrm{up}}\left(\vec{R}_{i}(k \Delta)\right)\right]
\end{aligned}
$$

where we used the density of unoccupied sites,

$$
\hat{n}_{\mathrm{up}}(\vec{r})=\sum_{i}^{N_{a}} \sum_{k=0}^{M_{a}-1} \delta\left(\vec{r}-\vec{R}_{i}(k \Delta)\right)-\hat{n}_{\mathrm{BI}}(\vec{r}) .
$$

The changes in model A due to Eq. (12) lead to a shift of $H_{\text {chain }}$ in Eq. (A15),

$$
\widetilde{H}_{\text {chain }}=H_{\text {chain }}+\int_{0}^{Z_{a}} d t \sum_{k=0}^{M_{a}-1} \delta(t-k \Delta) \omega_{\text {up }}\left(\vec{R}_{i}(t)\right),
$$

and a correction term for the free energy in Eq. (A13),

$$
\beta F_{g}=-\int d^{3} r \omega_{\mathrm{up}}(r)\left[n_{\mathrm{up}}(r)+n_{\mathrm{BI}}(r)\right] .
$$

Thus, two additional mean-field equations in Eq. (A19) are given by

$$
\delta n_{\mathrm{up}}: \omega_{\mathrm{up}}(\vec{x})-\beta \frac{\partial V}{\partial n_{\mathrm{up}}}=0
$$

and

$$
\delta \omega_{\mathrm{up}}: n_{\mathrm{up}}+n_{\mathrm{BI}}=\frac{N_{a}}{Q_{a} V} \sum_{k=0}^{M_{a}-1} q_{a}(\vec{x}, k \Delta) q_{a}\left(\vec{x}, Z_{a}-k \Delta\right) .
$$

Furthermore, $\widetilde{H}_{\text {chain }}$ changes $\delta F / \delta n_{\mathrm{BI}}=0$ in Eq. (A19) to

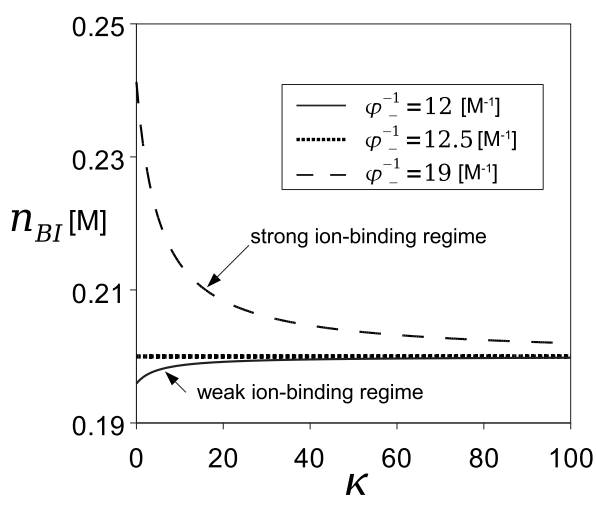

FIG. 5. $n_{\mathrm{BI}}$ as a function of $\kappa \cdot M_{a} / Z_{a}=0.5, g=0.08[\mathrm{M}]$, and $n_{a}=0.8[\mathrm{M}]$ are used. The $\kappa$-independent line is analytically obtained from $\varphi_{-}^{-1}=g^{-1}$. It should be noted that $\varphi_{-}^{-1}$ indicates the strength of monocomplexation denoted by Eq. (2), $K_{\text {eq}}$.

$$
\delta n_{\mathrm{BI}}: \frac{-\eta(\vec{x}) \nu_{\mathrm{BI}}}{\rho_{0}}+\omega_{\mathrm{BI}}(\vec{x})-q_{-} \beta \psi(\vec{x})+\omega_{\mathrm{up}}(\vec{x})-\beta \frac{\partial V}{\partial n_{\mathrm{BI}}}=0 .
$$

\section{B. Binding isotherm of model B}

In this section, uniform solutions to the modified meanfield equations are discussed. The integrated propagator in model $\mathrm{B}$ is written as

$$
q_{a}\left(Z_{a}\right)=e^{-\omega_{a} Z_{a}}\left(e^{K}+1\right)^{M_{a}-M_{a} \omega_{\mathrm{up}} .}
$$

The number of adsorbed molecules is then given by

$$
n_{\mathrm{BI}}=\frac{\gamma g \exp \left[\beta\left(\frac{\partial V_{\text {gel }}}{\partial n_{\text {up }}}-\frac{\partial V_{\text {gel }}}{\partial n_{\mathrm{BI}}}\right)\right]}{g \exp \left[\beta\left(\frac{\partial V_{\text {gel }}}{\partial n_{\text {up }}}-\frac{\partial V_{\text {gel }}}{\partial n_{\mathrm{BI}}}\right)\right]+\varphi_{-}}
$$

and

$$
n_{\mathrm{up}}=\gamma-n_{\mathrm{BI}} .
$$

Therefore, the potential form of Eq. (8) leads to

$$
n_{\mathrm{BI}}=\frac{g \gamma}{g+\varphi_{-} \exp \left[\kappa\left(2 n_{\mathrm{BI}}-\gamma\right)\right]},
$$

which is a self-consistent equation replacing Eq. (B2). For given concentrations of polymers and binding sites $(g$ and $\gamma$ ), $n_{\mathrm{BI}}$ can be determined by two parameters, $\varphi_{-}$and $\kappa$, for the interactions of the single-ion binding and gelation. An analytical solution of Eq. (22) is found to be $n_{\mathrm{BI}}^{*}=\gamma / 2$ with $\varphi_{-}^{-1}=g^{-1} \equiv \varphi_{-}^{*-1}$, which is independent of $\kappa$. This implies that $50 \%$ of total binding sites per chain are occupied. In the case of $\varphi_{-}^{-1}<\varphi_{-}^{*-1}, n_{\mathrm{BI}}$ increases with increasing $\kappa$, as shown in Fig. 5. Thus, the energy gain due to the gelation induces further adsorption of adsorbates. On the other hand, $n_{\mathrm{BI}}$ is reduced with increasing $\kappa$ in the case of $\varphi_{-}^{-1}>\varphi_{-}^{*-1}$. As shown in Eq. (B5), $\varphi_{-}^{-1}$ indicates the strength of binding adsorbates. When the value exceeds $\varphi_{-}^{*-1}$, more than $50 \%$ of binding sites per chain are occupied by adsorbed molecules. In this case, the number of unoccupied sites is relatively small. Therefore, the cross-linking formation drives the ad- 


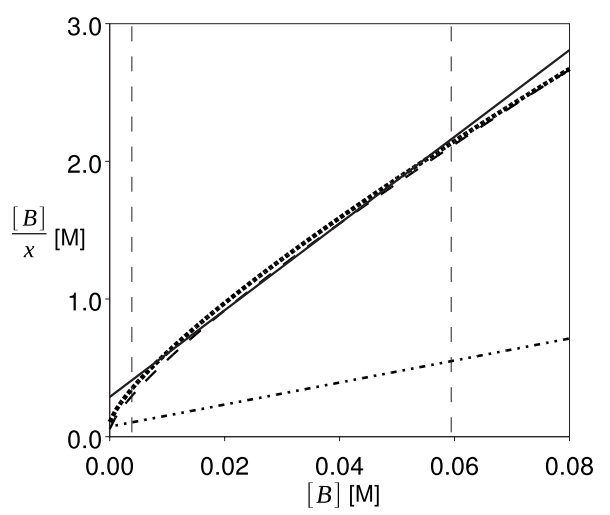

FIG. 6. The binding isotherm of PVA and borate in dilute aqueous solution with $\left(n_{a}, M_{a} / Z_{a}, \kappa, \varphi_{-}^{-1}\right)=\left(3[\mathrm{M}], 0.5,4.1,0.04\left[\mathrm{M}^{-1}\right]\right)$ and $(15[\mathrm{M}], 0.5,1$, $\left.0.02\left[\mathrm{M}^{-1}\right]\right)$. The value of $M_{a} / Z_{a}$ in Ref. 3 is used. The solid straight line is reproduced from the linear fitting of the experimental observations in Ref. 3. The slightly curving dashed lines are our theoretical results. The dot-dashed line below the curved lines is obtained using the standard Langmuir adsorption equation [Eqs. (2) and (B7)] with $M_{a} / Z_{a}=0.5$. The experiment is performed at concentrations within the boundary denoted by the vertical dashed lines. Note that the theory predicts a deviation from the linear fitting outside the region.

sorbed molecules to be released into a bulk phase, leading to a decrease in $n_{\mathrm{BI}}$.

\section{Binding isotherm of an intraconnected polymer}

In dilute aqueous solution with borate ions, single PVA polymer chains are largely isolated. Their conformation can collapse into a dense state via intrachain bridges due to cross-linking formation. ${ }^{3,8}$ To evaluate this state in the theory, the MFA is applied to a globule in which the segment distribution is assumed to be uniform, as discussed in Ref. 35. That is, we consider the intraconnected chain as an approximately homogeneous globule in this study. Equation (22) is then used to calculate the binding isotherm of this system. The results are shown in Fig. 6. In the case that the radius of gyration of a PVA molecule is approximated using the scaling relation of a self-avoiding walk, $R_{g}$ $=0.4205 N^{0.5934} b_{\text {kuhn }}$, the segment density is estimated at $n_{a}$ $\sim 0.2[\mathrm{M}]{ }^{36}$ Therefore, $n_{a}$ is set to be larger than $0.2[\mathrm{M}]$ but smaller than the density of a completely collapsed globule, $15[\mathrm{M}]$. In the range of these concentrations, we obtain qualitative agreement with the experiment using typical values of $\kappa \cdot{ }^{17}$

\section{Sol-gel transition}

In experiments, the formation of a homogeneous gel is often observed upon addition of adsorbate molecules. ${ }^{6,12,17} \mathrm{In}$ aqueous solution of PVA and borate ions, the gel demixes with increasing borate ion concentrations, and then further addition of borate ions induces a homogeneous phase of the gel again. In this case, largely deformed spinodal curves are observed, as derived from the model on a mean-field level in Sec. II E. In this section, the sol-gel transition is evaluated within the scope of the MFA.

The mean number of cross-linking formations is given by (the total energy of the cross-linking formations)/ (the didiol-borate complexation energy per dicomplex). The

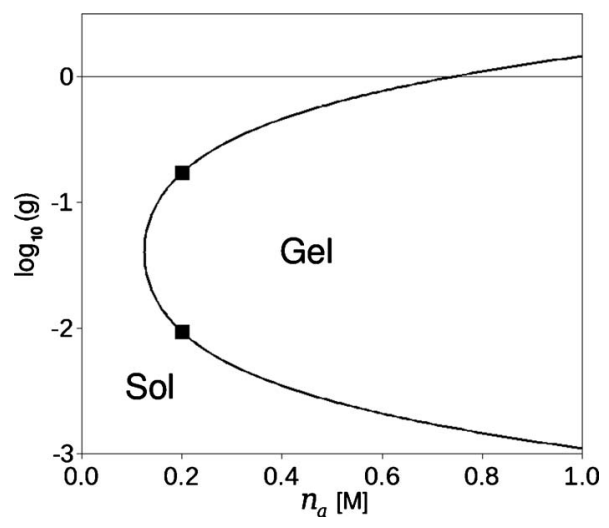

FIG. 7. The boundary of the sol-gel transition with $\left(M_{a} / Z_{a}, \kappa, \varphi_{-}^{-1}, K_{d}\right)$ $=\left(0.1,2,25\left[\mathrm{M}^{-1}\right]\right.$, and $\left.1\left[\mathrm{M}^{-1}\right]\right)$. Note that the $y$-axis for $g$ is scaled by $\log _{10}$. The sol-gel transition occurs inside the line. The squares are shown for a guide to the eye. They correspond to those in Fig. 8.

gelation occurs at a threshold of the concentration where a pair of polymer chain provides a single cross-linking formation. ${ }^{35}$ Therefore, Eqs. (8) and (9) lead to a criterion of gelation,

$$
\frac{\kappa}{E} n_{\mathrm{BI}} n_{\mathrm{up}} \geq \frac{n_{a}}{2}
$$

Thus, the critical degree of conversion (or gel point) is obtained by solving Eqs. (22) and (23). In Fig. 7, the gelation is shown to arise from the sol with increasing borate concentrations. Upon further addition of borate ions, however, the model predicts a re-entrance into a sol phase. Figure 8 indicates that the number of unoccupied binding sites is reduced to $<25 \%$ with the borate concentrations across the upper boundary (the square) in Fig. 7. Thus, the dicomplex formation (PVA-borate-PVA) becomes rather difficult in this regime.

The results of the sol-gel transition with experimental conditions of PVA and borate in aqueous solution are shown in Fig. 9. Overall behaviors of the transition in the theory and experiment are in agreement in the context of the MFA. ${ }^{17}$ In particular, the theoretical result leads to a crossing of the spinodal and sol-gel transition lines at at $n_{a} \sim 0.6[\mathrm{M}]$, as observed in the experiment.

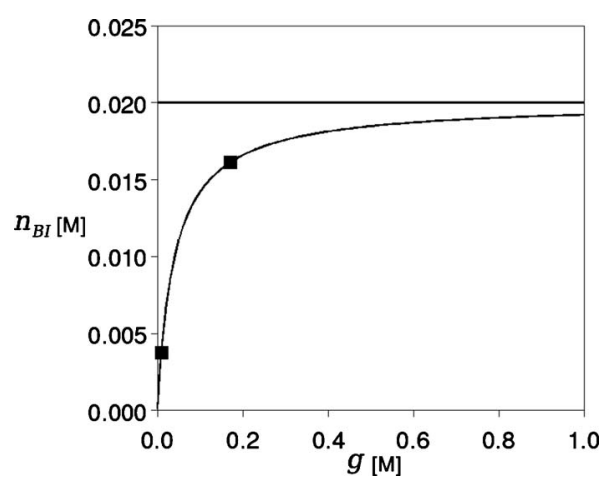

FIG. 8. $\quad n_{\mathrm{BI}} \quad$ with $\left(M_{a} / Z_{a}, \kappa, \varphi_{-}^{-1}, K_{d}, n_{a}\right)=\left(0.1,2,25\left[\mathrm{M}^{-1}\right], 1\right.$ $\left[\mathrm{M}^{-1}\right]$, and $\left.0.2[\mathrm{M}]\right)$. The asymptotic solution to Eq. (22), $n_{\mathrm{BI}}=\gamma$, is indicated as a horizontal line. The parameters in Fig. 7 are also used here. Note that the values on the squares correspond to those in Fig. 7. 


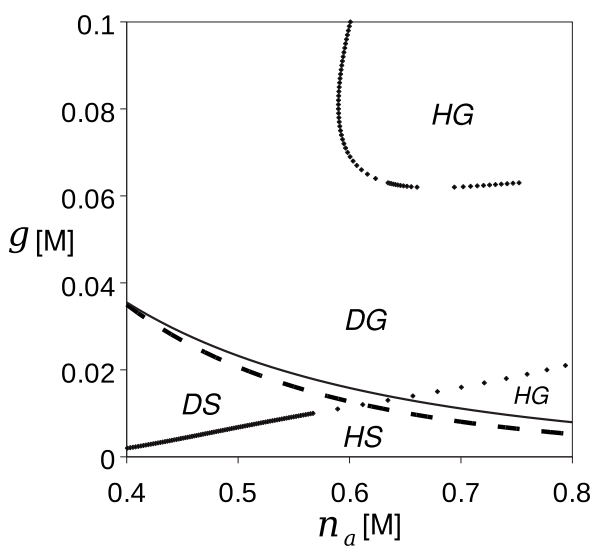

FIG. 9. The lower boundary of the sol-gel transition. The solid and dashed lines are obtained using $\left(M_{a} / Z_{a}, \kappa, \varphi_{-}^{-1}, K_{d}\right)$ $=\left(0.5,4,0.04\left[\mathrm{M}^{-1}\right]\right.$, and $\left.\left.1\left[\mathrm{M}^{-1}\right]\right)\right)$ and $\left(0.5, \quad 6, \quad 0.08\left[\mathrm{M}^{-1}\right]\right.$, $\left.0.35\left[\mathrm{M}^{-1}\right]\right)$, respectively. In experiments, $\kappa \sim 1$ and $K_{d} \sim 0.35\left[\mathrm{M}^{-1}\right]$ are estimated (Ref. 17). The isotacticity of $-\mathrm{OH}$ groups is set to be $50 \%$ in diad. The upper dotted lines are the spinodal lines given in Fig. 4. (1) HG: homogeneous gel; (2) HS: homogeneous sol; (3) DG: demixed gel; and (4) DS: demixed sol.

\section{DISCUSSION AND CONCLUSIONS}

In this paper, a SCFT of polymer-ionic molecule complexation is developed. Adsorption of ionic molecules typically leads to a competition between enthalpy gain and entropy loss in a free energy, providing a large driving force for phase instabilities.

An Ising-like binding variable, Eq. (3), is introduced to describe the number of adsorbed molecules through specificbinding interactions. Closed-loop spinodal lines for instability of homogeneous phase are shown to exist in the model, where the closed structure of the phase diagrams is induced by a repulsive interaction in the free energy, Eq. (5), due to a single occupancy on each binding site.

In many experiments, the complexation leads to a gelation through cross-linking formations, which also correlates with binding isotherms of adsorbates. To evaluate the effect, a mean-field interaction potential, Eq. (8), is included in the model. The correlation between occupied and unoccupied binding sites gives a modified equation for the binding isotherm, Eq. (22). In this paper, uniform solutions to meanfield equations are studied. Therefore, the effect of the inhomogeneity of polymer conformations induced by adsorbed adsorbates is not included in Eq. (22). The equilibrium constant of single-ion bindings leads to three types of solutions to Eq. (22) as a function of the parameter for the strength of cross-links, $\kappa$. The results indicate that polymers overcrowded with adsorbates release them from the binding sites in order to gain cross-linking formations.

The model is applied to binding isotherms of a model system, PVA (or HPG) with borate ions in aqueous solution. Spinodal lines of the system with low concentrations of salts are also discussed. In these cases, we obtained qualitative agreements between the theory and experiments. Furthermore, the sol-gel transition of PVA with borate ions is evaluated using Eqs. (22) and (23). The results qualitatively agree with an experimental observation. In this study, the Debye screening effect is not assessed because the model is used for a system with a weak ionic strength. In particular, the effect of specific-binding interactions on the miscibility of the polymers is mainly evaluated. We also remark that the charge screening may straightforwardly be derived from the model by the random phase approximation, as discussed in Ref. 28 .

In the present model, the adsorption of monovalent ionic molecules is discussed. In general, however, multivalent ionic molecules can be bound to adsorbents under specific conditions. For example, a single polyelectrolyte with the adsorption of both monovalent and divalent ions is studied by Kundagrami and Muthukumar. ${ }^{33,34}$ In this case, different ionic species are adsorbed on the binding sites of a polyelectrolyte chain. We remark that the adsorption of multivalent ions can also be described using more binding variables in our model.

\section{ACKNOWLEDGMENTS}

We would like to thank Dr. Robert Pelton and Mr. Yuguo Cui for many useful discussions on labile polymers. This work was supported by the Natural Sciences and Engineering Research Council of Canada (NSERC).

\section{APPENDIX A: DERIVATION OF MODEL A}

The inhomogeneous densities of the molecules are written as

$$
\begin{aligned}
& \widehat{n_{j}}=\sum_{i}^{N_{j}} \delta\left(\vec{r}-\vec{r}_{i}^{(j)}\right), \\
& \hat{n}_{-}=\sum_{i}^{N_{-}-N_{\mathrm{BI}}} \delta\left(\vec{r}-\vec{r}_{i}^{(-)}\right), \\
& \hat{n}_{\mathrm{BI}}=\sum_{i}^{N_{a}} \sum_{k}^{M_{a}-1} C_{i}^{(a)}(k \Delta) \delta\left(\vec{r}-\vec{R}_{i}^{(a)}(k \Delta)\right), \\
& \hat{n}_{a}=\sum_{i}^{N_{a}} \int_{0}^{Z_{a}} d t \delta\left(\vec{r}-\vec{R}_{i}^{(a)}(t)\right), \\
& \widehat{n_{v}}=\sum_{i}^{N_{v}} \delta\left(\vec{r}-\vec{r}_{i}^{(v)}\right),
\end{aligned}
$$

where $\widehat{n}_{j}, \hat{n}_{-}, \hat{n}_{\mathrm{BI}}, \hat{n}_{a}$, and $\hat{n}_{v}$ are the densities of the salt ion, free nonadsorbed ionic molecule, adsorbed ionic molecule, polymer, and solvent, respectively. $\vec{R}_{i}^{(a)}(t)$ is the $t$ th position of monomers on the $i$ th polymer $\left(t=0, \ldots, Z_{a}\right), \vec{r}_{i}^{(k)}$ is the $i$ th position of the $k$ species, and $Z_{a}$ denotes the number of statistical segments. A set of parameters, $v_{s}$, is defined to characterize the hard core volumes of monomers and molecules. We use the convention that an index, $s$, runs over all species $(j, v,-, \mathrm{BI}$ and $a)$. A reference density, $\rho_{0}=1 / v_{v}$, and the 
ratios of the volumes to a reference volume, $\nu_{s}=v_{s} / v_{v}$, are used. The incompressibility of the system, $\Sigma_{s} \nu_{s} \hat{n}_{s}(\vec{r}) / \rho_{0}=1$, is assumed. In this paper, flexible polymer chains are studied, and hence a continuous Gaussian chain model is adopted. The Gaussian stretching energy of the polymers is given by

$$
H_{0}=\frac{3}{2 b_{a}^{2}} \sum_{i}^{N_{a}} \int_{0}^{Z_{a}} d t\left(\frac{d \vec{R}_{i}(t)}{d t}\right)^{2}
$$

where $b_{a}$ denotes a statistical segment length. The binding energy between ionic molecule and binding site is expressed as $\varepsilon_{b}=-\beta \varepsilon_{0}<0$. Thus, the total adsorption energy is $H_{\mathrm{ad}}$ $=\varepsilon_{b} N_{\mathrm{BI}}$. The electrostatic interaction between charged molecules is written as

$$
H_{e}=\frac{1}{2} \int d^{3} r \hat{\rho}(\vec{r}) v\left(r-r^{\prime}\right) \hat{\rho}\left(\vec{r}^{\prime}\right)
$$

where

$$
\hat{\rho}(\vec{r})=\sum_{j}^{I} q_{j} \hat{n}_{j}+q_{-} \hat{n}_{-}+q_{-} \hat{n}_{\mathrm{BI}}
$$

and

$$
v(r)=l_{B} / r
$$

$l_{B} \equiv \beta e^{2} /\left(4 \pi \epsilon_{0}\right)$ is the Bjerrum length at which a thermal energy is comparable to the electrostatic interaction between two unit charges. $q_{j}$ and $q_{-}$are used to describe the valences of the charges for the salt ions and ionic molecule. Note that the noncomplexed polymers are neutral. However, adsorbed charged molecules convert them to polyelectrolytes. The interaction between segments is modeled as a standard form,

$$
\beta V\left(\hat{n}_{s}\right)=\lambda \int d^{3} r \hat{n}_{a}(\vec{r}) \hat{n}_{v}(\vec{r})
$$

The partition function can then be written as

$$
\begin{aligned}
Z= & \int \prod_{i}^{N_{a}} \mathcal{D} C_{i}^{(a)}(k \Delta) \mathcal{D} \vec{R}_{i}(t) \prod_{y=1}^{N_{v}} d \vec{r}_{y}^{(v)} \prod_{j}^{I} \prod_{l}^{N_{j}} d \vec{r}_{l}^{(j)} \prod_{m}^{N_{-}-N_{\mathrm{BI}}} d \vec{r}_{m}^{(-)} \\
& \times \prod_{r} \delta\left(\sum_{s} \frac{\nu_{s} \hat{n}_{s}(\vec{r})}{\rho_{0}}-1\right) \frac{\xi_{(-)}^{N_{-}-N_{\mathrm{BI}}}}{\left(N_{-}-N_{\mathrm{BI}}\right) !} \prod_{j}^{I} \frac{\xi_{j}^{N_{j}}}{N_{j} !} \frac{\xi_{a}^{N_{a}}}{N_{a} ! N_{v} !} \\
& \times e^{-\left(H_{0}+H_{\mathrm{ad}}+H_{e}+\beta V\left(\hat{n}_{s}\right)\right)},
\end{aligned}
$$

where $\xi_{s}$ denotes a single-molecular partition function due to the kinetic energy. Since a thermodynamic number of ionic molecules are contained in the model, $\left(N_{-}-N_{\mathrm{BI}}\right) \gg 0$ is used. The Hubbard-Stratonovich transformation and the Stirling's formula are then applied to Eq. (A11), leading to ${ }^{28}$

$$
Z=\int \mathcal{D} \psi \mathcal{D} \eta \prod_{s} \mathcal{D} n_{s} \mathcal{D} \omega_{s} e^{-\beta F}
$$

The free energy functional of the system is given by

$$
\begin{aligned}
\beta F= & \int d^{3} r \eta(\vec{r})\left(\sum_{s} \frac{\nu_{s} n_{s}(\vec{r})}{\rho_{0}}-1\right)-\int d^{3} r \frac{1}{8 \pi l_{B}}(\nabla \psi)^{2} \\
& +\beta V\left(n_{s}\right)-\int d^{3} r \sum_{s} \omega_{s}(\vec{r}) n_{s}(\vec{r})+\int d^{3} r \psi(\vec{r}) \\
& \times\left(\sum_{j} q_{j} n_{j}(\vec{r})+q_{-} n_{-}(\vec{r})+q_{-} n_{\mathrm{BI}}(\vec{r})\right)-N_{v} \ln \int d^{3} r e^{-\omega_{v}} \\
& -\sum_{s} N_{j} \ln \int d^{3} r e^{-\omega_{j}-N_{-}} \ln \int d^{3} r e^{-\omega_{-}-N_{a} \ln \left(V Q_{a}\right)} \\
& -\ln \left(\frac{\xi_{(-)}^{N_{-}}}{N_{-} !} \prod_{j}^{I} \frac{\xi_{j}^{N_{j}}}{N_{j} !} \frac{\xi_{a}^{N_{a}}}{N_{a} !} \frac{\xi_{v}^{N_{v}}}{N_{v} !}\right)
\end{aligned}
$$

where

$$
Q_{a}=\frac{1}{V} \int \mathcal{D} \vec{R}(t) e^{-H_{\text {chain }}}=\frac{1}{V} \int d \vec{r} q_{a}\left(\vec{r}, Z_{a}\right),
$$

$$
\begin{aligned}
H_{\text {chain }}= & \int_{0}^{Z_{a}} d t\left[\frac{3}{2 b_{a}^{2}}\left(\frac{d \vec{R}}{d t}\right)^{2}+\omega_{a}(\vec{R}(t))\right. \\
& \left.-\sum_{k}^{M_{a}-1} \delta(t-k \Delta) \ln \left(1+e^{K(\vec{R}(t))}\right)\right],
\end{aligned}
$$

and

$$
K(\vec{R}(t))=\ln \left(\frac{N_{-} e^{-\varepsilon_{b}}}{\xi_{(-)}}\right)-\ln \left(\int d^{3} r e^{-\omega_{-}(\vec{r})}\right)-\omega_{\mathrm{BI}}(\vec{R}(t)) .
$$

The integrated propagator, $q_{a}$, is the solution to the modified diffusion equation, ${ }^{37}$

$$
\frac{\partial q_{a}(\vec{r}, t)}{\partial t}=\frac{b_{a}^{2}}{6} \nabla^{2} q_{a}(\vec{r}, t)-\omega_{a}^{\mathrm{eff}}(\vec{r}, t) q_{a}(\vec{r}, t),
$$

where

$$
\omega_{a}^{\mathrm{eff}}=\omega_{a}(\vec{r})-\sum_{k}^{M_{a}-1} \delta(t-k \Delta) \ln \left(1+e^{K(\vec{R}(t))}\right)
$$

Mean-field equations are obtained by the minimization of the free energy functional, Eq. (A13), with respect to functional variables. This leads to the following selfconsistent equations:

$$
\begin{gathered}
\delta n_{j}: \frac{-\eta(\vec{x}) \nu_{j}}{\rho_{0}}+\omega_{j}(\vec{x})-q_{j} \beta \psi(\vec{x})=0, \\
\delta n_{-}: \frac{-\eta(\vec{x}) \nu_{-}}{\rho_{0}}+\omega_{-}(\vec{x})-q_{-} \beta \psi(\vec{x})=0, \\
\delta n_{\mathrm{BI}}: \frac{-\eta(\vec{x}) \nu_{\mathrm{BI}}}{\rho_{0}}+\omega_{\mathrm{BI}}(\vec{x})-q_{-} \beta \psi(\vec{x})=0, \\
\delta n_{v}: \frac{-\eta(\vec{x}) \nu_{v}}{\rho_{0}}+\omega_{v}(\vec{x})-\beta \frac{\delta V}{\delta n_{v}}=0,
\end{gathered}
$$




$$
\begin{aligned}
& \delta n_{a}: \frac{-\eta(\vec{x}) \nu_{a}}{\rho_{0}}+\omega_{a}(\vec{x})-\beta \frac{\delta V}{\delta n_{a}}=0, \\
& \delta \eta: \sum_{s} \frac{-n_{s}(\vec{x}) \nu_{s}}{\rho_{0}}+1=0, \\
& \delta \omega_{j}: n_{j}(\vec{x})-\frac{N_{j} e^{-\omega_{j}(\vec{x})}}{\int d^{3} r e^{-\omega_{j}(\vec{r})}}=0, \\
& \delta \omega_{-}: n_{-}(\vec{x})-\frac{N_{-} e^{-\omega_{-}(\vec{x})}}{\int d^{3} r e^{-\omega_{-}(\vec{r})}} \\
& \quad+\frac{1}{V Q_{a}} \sum_{k}^{M_{a}-1} \int^{\mathcal{D} R}(k \Delta) q_{a}(\vec{R}(k \Delta), k \Delta) q_{a}\left(\vec{R}(k \Delta), Z_{a}-k \Delta\right) \\
& \quad \times \frac{e^{K(\vec{R}(K \Delta))-\omega_{-}(\vec{x})}}{\left(1+e^{K(\vec{R}(K \Delta))}\right) \int d^{3} r e^{-\omega_{-}(\vec{r})}}=0, \\
& \delta \omega_{v}: n_{v}(\vec{x})-\frac{N_{v} e^{-\omega_{v}(\vec{x})}}{\int d^{3} r e^{-\omega_{v}(\vec{r})}}=0, \\
& \delta \omega_{\mathrm{BI}}: n_{\mathrm{BI}}(\vec{x})-\frac{1}{V Q_{a}} \sum_{k}(\vec{x})-\frac{1}{V Q_{a}} \int_{0}^{Z_{a}} q_{a}(\vec{x}, k \Delta) q_{a}\left(\vec{x}, Z_{a}-k \Delta\right) \frac{e^{K(\vec{x})}}{\left(1+e^{K(\vec{x})}\right)} \\
& =0, \quad q_{a}(\vec{x}, t) q_{a}\left(\vec{x}, Z_{a}-t\right)=0,
\end{aligned}
$$

\section{APPENDIX B: THE FREE ENERGY OF THE HOMOGENEOUS PHASE WITHOUT POLYMER-ION CROSS-LINKING}

The self-consistent equations, Eq. (A19), are applied to a homogeneous phase. The position dependence in the equations is then ignored, and thus we obtain

$$
q_{a}(k \Delta) q_{a}\left(Z_{a}-k \Delta\right)=q_{a}\left(Z_{a}\right)=e^{-\omega_{a} Z_{a}}\left(e^{K}+1\right)^{M_{a}}
$$

and

$$
n_{\mathrm{BI}}=\frac{g \gamma}{\left(g+\varphi_{-}\right)}
$$

where

$$
\begin{aligned}
& K=\ln \left(\frac{N_{-}}{V \xi_{(-)}} e^{-\varepsilon_{b}}\right)+\omega_{-}-\omega_{\mathrm{BI}}, \\
& g=\frac{N_{-}}{V}, \\
& \varphi_{-}=\xi_{(-)} e^{\varepsilon_{b}},
\end{aligned}
$$

$$
\gamma=\frac{M_{a} N_{a}}{V}=\left(\frac{M_{a}}{Z_{a}}\right) n_{a} .
$$

$g$ and $\gamma$ indicate the overall concentrations of adsorbate molecules and binding sites, respectively. The equilibrium constant, $K_{\text {eq }}$, of Eq. (2) is calculated by

$$
K_{\mathrm{eq}}=\frac{n_{\mathrm{BI}}}{n_{-}\left(\gamma-n_{\mathrm{BI}}\right)} \text {. }
$$

Equation (B7) with $g \gg \gamma$ then leads to

$$
K_{\mathrm{eq}}=\varphi_{-}^{-1}
$$

In this case, the free energy (Eq. (A13)) in homogeneous phase can be written as

$$
\begin{aligned}
\frac{\beta F}{V}= & \frac{\beta V\left(n_{a}, n_{v}\right)}{V}-\frac{M_{a} n_{a}}{Z_{a}} \ln \left(1+g K_{\mathrm{eq}}\right)+\frac{n_{a}}{Z_{a}} \ln n_{a}+g \ln g \\
& +\sum_{j} n_{j} \ln n_{j}+n_{v} \ln n_{v} .
\end{aligned}
$$

${ }^{1}$ T. P. Lodge, Science 321, 50 (2008).

${ }^{2}$ H. Nishikawa and E. Tsuchida, J. Phys. Chem. 79, 2072 (1975).

${ }^{3}$ H. Ochiai, Y. Fujino, Y. Tadokoro, and I. Murakami, Polymer 21, 485 (1980).

${ }^{4}$ H. Ochiai, Y. Fujino, Y. Tadokoro, and I. Murakami, Polym. J. (Tokyo, Jpn.) 14, 423 (1982).

${ }^{5}$ L. Leibler, E. Pezron, and P. A. Pincus, Polymer 29, 1105 (1988).

${ }^{6}$ E. Pezron, A. Ricard, F. Lafuma, and R. Audebert, Macromolecules 21, 1121 (1988).

${ }^{7}$ J. C. Ma and D. A. Dougherty, Chem. Rev. (Washington, D.C.) 97, 1303 (1997).

${ }^{8}$ C. Y. Chen, J. Y. Guo, T. L. Yu, and S. C. Wu, J. Polym. Res. 5, 67 (1998).

${ }^{9}$ Y. Tran and P. Auroy, Eur. Phys. J. E 5, 65 (2001).

${ }^{10}$ Y. G. Cui, R. Pelton, and H. Ketelson, Macromolecules 41, 8198 (2008).

${ }^{11}$ M. Shibayama, H. Yoshizawa, H. Kurokawa, H. Fujiwara, and S. Nomura, Polymer 29, 2066 (1988).

${ }^{12}$ E. Pezron, L. Leibler, A. Ricard, and R. Audebert, Macromolecules 21, 1126 (1988).

${ }^{13}$ M. A. V. Axelos, M. M. Mestdagh, and J. Francois, Macromolecules 27, 6594 (1994).

${ }^{14}$ C. Lu, L. Kostanski, H. Ketelson, D. Meadows, and R. Pelton, Langmuir 21, 10032 (2005).

${ }^{15}$ M. M. Coleman, J. F. Graf, and P. C. Painter, Specific Interactions and the Miscibility of Polymer Blends (Technomic, Lancaster, 1991).

${ }^{16}$ H. Kurokawa, M. Shibayama, T. Ishimaru, S. Nomura, and W. I. Wu, Polymer 33, 2182 (1992).

${ }^{17}$ G. Keita, A. Ricard, R. Audebert, E. Pezron, and L. Leibler, Polymer 36, 49 (1995).

${ }^{18}$ A. G. Cherstvy, A. A. Kornyshev, and S. Leikin, J. Phys. Chem. B 106, 13362 (2002).

${ }^{19}$ M. Weck, Polym. Int. 56, 453 (2007).

${ }^{20}$ H. Xu, S. Srivastava, and V. A. Rotello, Adv. Polym. Sci. 207, 179 (2007).

${ }^{21}$ E. Carretti, S. Grassi, M. Cossalter, I. Natali, G. Caminati, R. G. Weiss, P. Baglioni, and L. G. Dei, Langmuir 25, 8656 (2009).

${ }^{22}$ Y. F. Wei and P. Y. Hsiao, J. Chem. Phys. 127, 064901 (2007).

${ }^{23}$ M. Muthukumar, J. Chem. Phys. 120, 9343 (2004).

${ }^{24}$ S. Liu and M. Muthukumar, J. Chem. Phys. 116, 9975 (2002).

${ }^{25}$ A. Y. Grosberg, T. T. Nguyen, and B. I. Shklovskii, Rev. Mod. Phys. 74, 329 (2002).

${ }^{26}$ T. T. Nguyen, A. Y. Grosberg, and B. I. Shklovskii, Phys. Rev. Lett. 85, $1568(2000)$.

${ }^{27}$ E. H. Feng, W. B. Lee, and G. H. Fredrickson, Macromolecules 40, 693 (2007). 
${ }^{28}$ A. C. Shi and J. Noolandi, Macromol. Theory Simul. 8, 214 (1999).

${ }^{29}$ Q. Wang, T. Taniguchi, and G. H. Fredrickson, J. Phys. Chem. B 108, 6733 (2004).

${ }^{30}$ I. Nakamura and A. C. Shi, Phys. Rev. E 80, 021112 (2009).

${ }^{31}$ H. T. Davis, Statistical Mechanics of Phases, Interfaces, and Thin Films (VCH, New York, 1996).

${ }^{32}$ N. A. Peppas and E. W. Merrill, J. Polym. Sci., Part A-1 14, 459 (1976).
${ }^{33}$ A. Kundagrami and M. Muthukumar, J. Chem. Phys. 128, 244901 (2008).

${ }^{34}$ A. Kundagrami and M. Muthukumar, Macromolecules 43, 2574 (2010).

${ }^{35}$ A. N. Semenov and M. Rubinstein, Macromolecules 31, 1373 (1998).

${ }^{36}$ I. Teraoka, Polymer Solutions (Wiley, New York, 2002).

${ }^{37}$ T. Kawakatsu, Statistical Physics of Polymers (Springer-Verlag, Berlin, 2004). 